\title{
Does broadband address the cycle skipping of full waveform inversion in complex areas?
}

\author{
Denes Vigh*, Xin Cheng, Kun Jiao, Zhen Xu and Wei Dai Schlumberger/WesternGeco
}

Copyright 2019, SBGf - Sociedade Brasileira de Geofísica

This paper was prepared for presentation during the $16^{\text {th }}$ International Congress of the Brazilian Geophysical Society held in Rio de Janeiro, Brazil, 19-22 August 2019.

Contents of this paper were reviewed by the Technical Committee of the $16^{\text {th }}$ International Congress of the Brazilian Geophysical Society and do not necessarily represent any position of the SBGf, its officers or members. Electronic reproduction or represent any postion of the SBG its oficers or members. Electronic reproduction or of the Brazilian Geophysical Society is prohibited.

Abstract

The conventional FWI is exposed to cycle skipping especially when the initial model is inadequate and the ultra-low frequencies are not present in the observed data. If the FWI does not have the power to mitigate the cycle skipping then initial velocity must be built by traveltime tomography. This way the model building would comprise of travel-time tomography followed by FWI and finish the flow with travel-time tomography. The industry is working towards how FWI would overcome on half wavelength convergence criteria in order to start the iterative model updates at higher avaiable frequency. Mitigting cycle skiping is even more imprtant when the reflection based $\mathrm{FWI}$ is employed to build the deep update in the model.Going beyond the conventional and reflection-based FWI's half wavelengths criteria allows us to employ FWI in the velocity model building flow even there is only relativley high starting frequnecies present in the data collection. If broad band data acquisition or processing avaible then FWI robustness is ensured.

\section{Introduction}

Full-waveform inversion (FWI) has emerged over the last decade as a high-end tool for high-resolution, complex model building. As a data-fitting algorithm, FWI exploits the full content of the recorded seismic waveform to derive subsurface earth models. Diving waves, pre-critical and post-critical reflections, and diffractions all carry different information about the subsurface. The oil and gas industry has seen very successful applications of FWI in different geological environments with various data acquisition methods. Usually, FWI works best with data acquisition techniques that can deliver long-offset and/or low-frequency seismic data to construct the deep parts of the subsurface and to mitigate the cycle-skip issue due to the nonlinear nature of $\mathrm{FWI}$. In the past decade, successful FWI applications have all used the early arrivals, diving waves, and refractions to successfully update the shallow parts of the model. The remaining question is how do we achieve meaningful deep updates beyond that which conventional FWI can provide using data-domain inversion rather than relying on imagedomain approaches such as ray-based tomography or wave-equation migration velocity analysis? This issue becomes significant when the data do not have long offsets and are dominated by reflections. To use reflection data to successfully update the low-wavenumber model in FWI we propose a method to decompose the model representation into a background model that governs the kinematics, and a reflectivity model that governs the dynamics of the wavefield.

\section{Seismic acquisition and FWI}

While the first convincing applications of 3D FWI were performed with stationary-receiver as Ocean Bottom Cable (OBC) geometry, (Plessix and Perkins, 2010; Sirgue et al., 2010), many applications are currently performed with towed-streamer data especially for exploration purpose. The drawback of such acquisition is the limited length of the recording device which prevent deep targets from being sampled by diving waves and post-critical reflections for FWI. This issue has been partly bypassed by adapting $\mathrm{FWI}$ to reflection data leading to the so-called reflection-based waveform inversion (RFWI).RFWI relies on the scale separation between the reflectivity and the velocity macromodel underlying classical reflection processing workflow based upon alternated migration and migration-based velocity analysis. To some extent, RFWI breaks down the original FWI idea of continuously sampling the wavenumber spectrum of the subsurface by exploiting the rich angular illumination provided by wide-aperture acquisitions such as surface permanent receiver acquisitions (OBC, OBN, land) (Pratt et al., 1996). Today, long-offset multicomponent node acquisitions emerge as an alternative to towed-streamer acquisition for deep water offshore subsalt exploration. These stationary-receiver acquisitions offer the necessary versatility to design full-azimuth ultralong offset acquisition that are suitable to sample the full targeted structures with a wide variety of wave types in term of propagation regime (transmission versus reflection), propagation direction. This provides an optimal setting to really exploit the resolution power of FWI and its ability to reconstruct all of the mechanical parameters that govern wave propagation.

The recovered wavelengths in FWI are heavily influenced by the subsurface, the local velocity, and the illumination angular aperture. For transmissions and refractions, the large illumination angle apertures facilitate the reconstructing the long-wavelength parts of velocity models. For reflections, only short-wavelength parts tend 
to be recovered by $\mathrm{FWI}$ due to the narrow range of reflection angle apertures. This explains why $\mathrm{FWI}$ recovers long-wavelength components only in shallow areas and its resolving area improves when longer-offset data and lower frequencies are present. Unfortunately for conventional streamer data, the maximum offset is usually limited to $9 \mathrm{~km}$, in which case FWI updates are limited to the shallow section, especially in deep-water environments. To achieve meaningful deep updates, reflection data must be incorporated.

\section{Method}

The recovered wavelengths in FWI are heavily influenced by the subsurface, the local velocity, and the illumination angular aperture. For transmissions and refractions, the large illumination angle apertures facilitate the reconstruction of the long-wavelength parts of velocity models. For reflections, only short-wavelength parts tend to be recovered by FWI due to the narrow range of reflection angle apertures. This explains why $\mathrm{FWI}$ recovers long-wavelength components only in shallow areas and its resolving area improves when longer-offset data and lower frequencies are present. Unfortunately for conventional streamer data, the maximum offset is usually limited to $8 \mathrm{~km}$, in which case FWI updates are limited to the shallow section, especially in deep-water environments. To achieve kinematic improvements in the image gathers in the deep updates, reflection data must be incorporated. The background velocity change would come from the low wavenumber part of the kernel which can be associated with the backscattering energy from the reflection data rather than the high wave number which is the migration operator. Obtaining the kinematic changes in the deep part of the model or in other words separation the low wave number from the high wave number in the kernel can be achieved through different approaches. The first one is the wavefield decomposition (Liu et al., 2011) method to separate the backscatter noise from the migration operator. The second one would be the energy norm concept by Rocha et al. (2016) to derive the energy inner product between two related wavefield that leads to separating the low and high wave number components of kernel. The third approach would be via Born modeling using background velocity field and reflectivity to obtain the previously described separation. $\mathrm{Xu}$ et al. (2012), Brossier et al. (2014) and Vigh et al. (2016) proposed a method to decompose the model representation into a background model that governs the kinematics, and a reflectivity model that governs the dynamics of the wavefield. During FWI iterations, both the background model and the reflectivity model are updated. In our proposed reflection-based FWI (RFWI) approach, long- and short-wavelength model updates are decoupled; the long-wavelength information contributes to background model updates while the short-wavelength content contributes to the reflectivity updates.

FWI performance depends upon the robustness of the separation especially when the background velocity is not accurate and the resolution of the updates in horizontal and vertical sense. The resolution is where the differences can be observed between these three approaches. In our field examples, we employ the above mentioned third method to execute the reflection FWI for deep velocity updates.

\section{Field Data Examples and Results}

The field example consists of a $2 \times 4$ linear wide-azimuth data set with approximately a 9.6-km maximum offset acquired in the Gulf of Mexico. The gun array, the shot depth, and the cable depth allowed us to record low frequencies of about 2.5 to $3.5 \mathrm{~Hz}$ in spite of the rich lowfrequency content, we elected to start the conventional FWI from $4 \mathrm{~Hz}$ because the adjustive (cycle-skipmitigated) option was employed from a 1D type of starting velocity field (Figure 1a). FWl's forward simulation can easily include the source and receiver ghosts so for the classical FWI does not require broadband processing. First, we used the early arrivals as input to FWI from wide-azimuth data. After 10 iterations at $4 \mathrm{~Hz}$, we increased the frequency to $6 \mathrm{~Hz}$ in the multiscale manner to achieve higher resolution to the velocity update with a further seven iterations. After the first two frequency bands using early arrivals for the FWI update (Figure 1c), the updates were limited to $2.7 \mathrm{~km}$, When the depth slice difference is interrogated between the starting and the updated model (Figures $1 \mathrm{~b}$ and 1d) the first obvious conclusion is that FWI picked up the small- and mediumscale high-velocity carbonate carapaces and the lowvelocity shale bodies from the simple starting velocity field.

To extend the updates to the deep part of the basins and under the salt, we switched to the RFWI. The input data was deghosted to enrich the low end of the spectra for the RFWI which was run through 12 iterations at $3.5 \mathrm{~Hz}$. In the deep part of the section, we separated the low and high wavenumbers such that the low wavenumbers contributed to the velocity field while the high wavenumbers contributed to the reflectivity field. This product of RFWI may be used as a quality control because it is proportional to the image. Success was benchmarked against the appearance of inter-shale reflectivity, base shale, and better continuity of Top Wilcox, Top Cretaceous structures and any underlying Mesozoic strata.

The deep update is demonstrated by subsalt improvements in terms of imaging and velocity update. The initial velocity field was fine-tuned using reflectionbased FWI to achieve the final updated velocity field (Figures $2 c$ and $2 \mathrm{~d}$ ). This was used to compare the initial image (Figure $2 \mathrm{a}$ ) with the final velocity image (Figures $2 \mathrm{a}$ and $2 b$ ). The deep part of the model at the cretaceous depth shows improvements in reflector continuity and focusing after the deep reflection RFWI updates in the subsalt area. Improvement in the Wilcox and the Top Cretaceous are striking in these examples that proves the successful application of reflection full-waveform inversion. 


\section{Conclusions}

Conventional FWI has the limitation of diving-wave penetration that can be a few $\mathrm{km}$ below the seafloor and is too shallow in a deep-water environment. To extend this, reflections should be considered with the correct sensitivity kernel. This enables FWI to update the deeper section. Using the reflections with broadband processing, we can update both the low- and the mediumwavenumber parts of the velocity field beyond the diving wave penetrating power. Enriching the low frequencies during the data processing ensure the successful subsalt updates.

\section{References}

Brossier, R., S. Operto, and J. Virieux, 2014, Velocity model building from seismic reflection data by full-waveform inversion: Geophysical Prospecting, 63.2, 354-367.

Liu, F., G. Zhang, S. Morton, and J. Leveille, 2011, An effective imaging condition for reverse time migration using wavefield decomposition. Geophysics, Vol. 76, No. 1, pp. S29-S39.

Plessix, R. E. and C. Perkins, 2010, Full waveform inversion of a deep water ocean bottom seismometer dataset. First Break, 28:71-78.

Pratt, R. G., Z-M. Song, P. Williamson, and M. Warner. 1996, Two-dimensional velocity models from wide-angle seismic data by wavefield inversion. Geophysical Journal International 124, no. 2, 323-340.

Rocha, D., N. Tanushey, and P. Sava, 2016, Acoustic wavefield imaging using energy norm. Geophysics, Vol. 81, No. 4, pp. S151-S163.

Sirgue, L., O. I. Barkved, J. Dellinger, J. Etgen, U. Albertin, and J. H. Kommedal, 2010, Full waveform inversion: the next leap forward in imaging at Valhall. First Break, 28:65-70.

Vigh, D., K. Jiao, X. Cheng, D. Sun, and W. Lewis, 2016, Earth-model building from shallow to deep with fullwaveform inversion. The Leading Edge, Vol. 35, No. 12, pp. 1025-1030.
Xu, S., F. Chen, G. Lambaré, Y. Zhang, and D. Wang, 2012 , Inversion on reflected seismic wave: $82^{\text {nd }}$ Annual International Meeting, SEG, Expanded Abstracts, 1-7. 

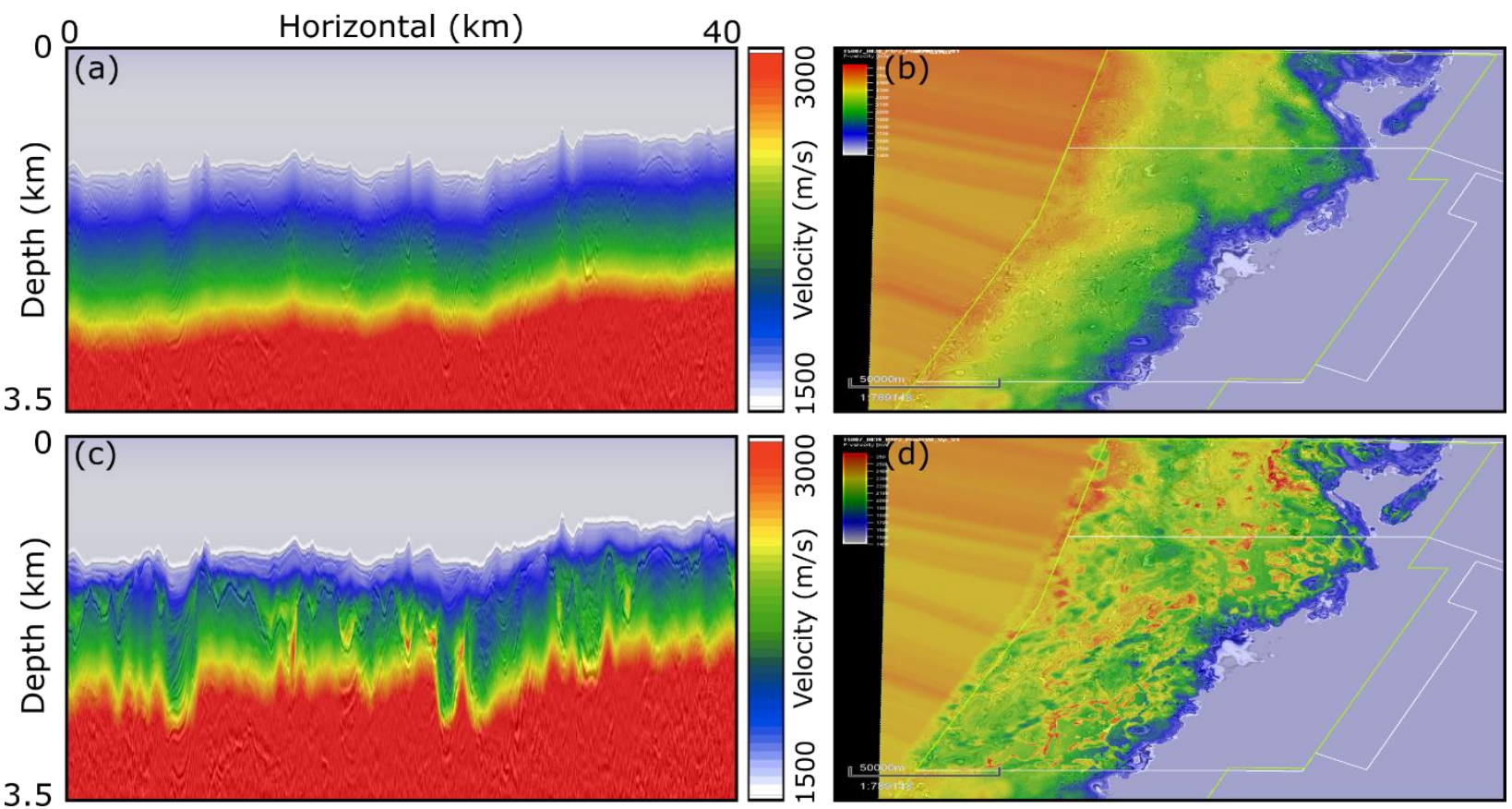

Figure 1 (a) Initial $1 D$ velocity model vertical section. (b) Initial $1 D$ velocity model depth slice at $2 \mathrm{~km}$. (c) FWI updated velocity after two frequency band updates in vertical section. (d) FWI updated velocity after two frequency band updates depth slice at $2 \mathrm{~km}$.
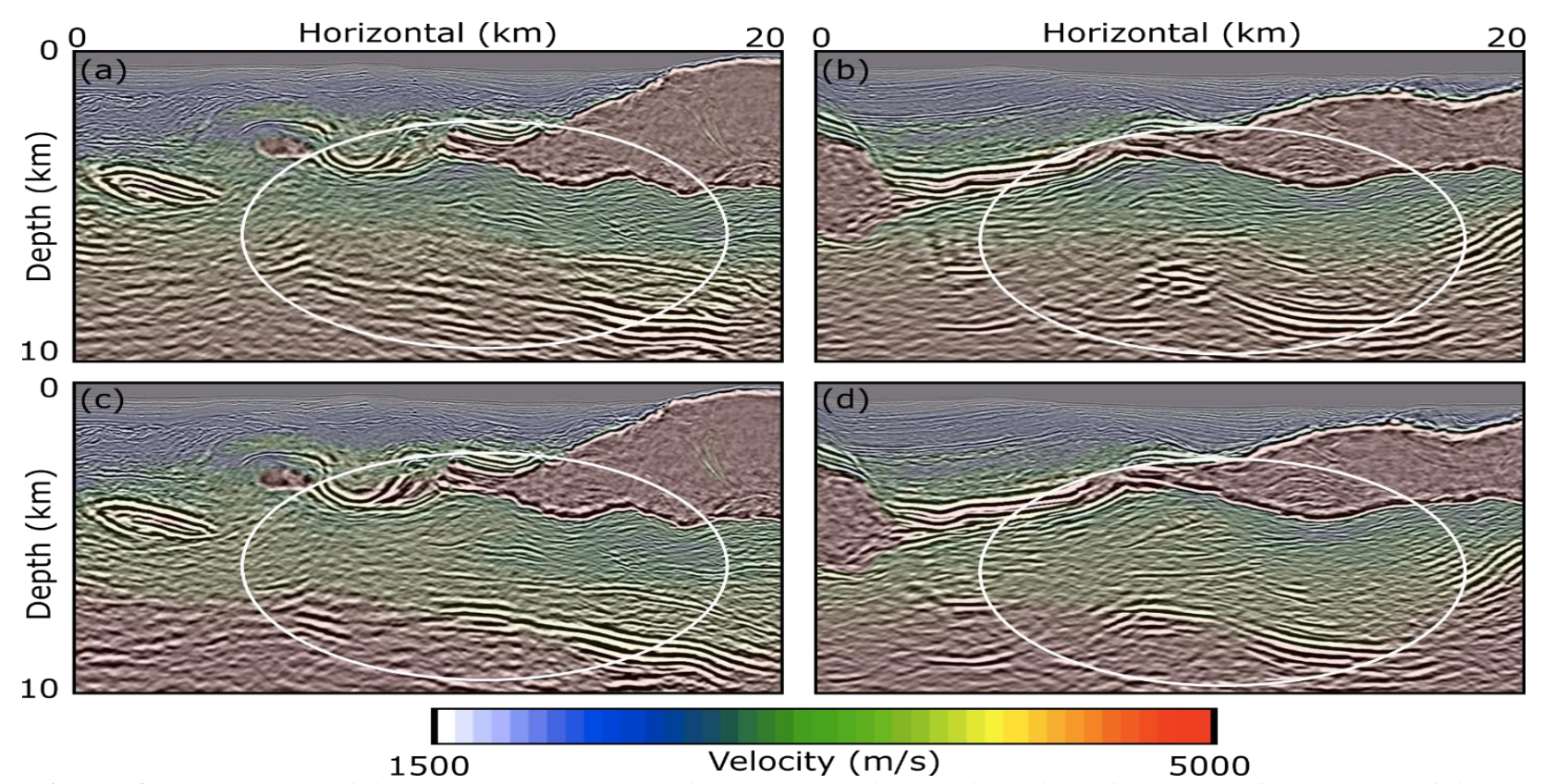

Figure 2 (a) Input model to reflection FWI with image overlay in the inline direction. (b) Input model to reflection FWI with image overlay in the crossline direction. (c) Reflection FWI updated model with image overlay in the inline direction. (d) Reflection FWI updated model with image overlay in the crossline direction. 